\title{
Intensive psychotherapy with in-patients at Chestnut Lodge Hospital
}

\author{
Marco C. Chiesa, Joint Senior Registrar in Psychotherapy, St Bernard's Wing, Ealing \\ Hospital and the Cassel Hospital, Richmond, Surrey
}

Last summer I spent two weeks as visiting psychiatrist at Chestnut Lodge Hospital, one of the pioneering institutions in the psychoanalytically oriented treatment of psychotic disorders. This approach has been modified over the years until the present time having been complemented by new developments in the social, psychological and biological fields of psychiatry.

\section{History}

Chestnut Lodge is a private psychiatric hospital in Rockville, Maryland - a suburban town located 20 miles north west of Washington DC. The hospital was founded in 1910 and soon adopted a psychodynamic approach to the treatment of psychosis. This was rather a brave and risky choice because in the same years Freud was expressing pessimistic views with regard to the suitability of psychoanalysis for psychotic patients who, he believed, had no capacity for transference, and a too weak and unintegrated ego to withstand the intense impulses and affects evoked by the analytical situation.

The work of Frieda Fromm-Reichmann, ${ }^{1}$ whose association with the hospital lasted for 22 years until her death in 1957, has been universally recognised as the most influential in shaping and consolidating the intensive in-patient psychotherapeutic approach to schizophrenia. She integrated the already existing developments into a relatively comprehensive body of theory and technique. Initially, she believed that psychotic patients experience serious traumas in early infancy, when their egos are at their most vulnerable, and when the infant lives grandiosely in a narcissistic world of his own. These traumatic experiences, generally felt to be inflicted by an insensitive and unempathic mother, render the child hypersensitive to later frustrations, from which the patient then withdraws into an autistic delusional world. The therapist's task is to recreate in his interaction with the patient a mother-infant relationship in which the therapist's endurance of the patient's continuous testing and retesting of his solidity and reliability would allow the patient to come out of his autistic world into a trusting relationship, leading to the development of a better contact with external reality.
From the technical point of view she recommended that the patient should neither lie on the couch nor be asked to give free associations. The analyst creates an atmosphere of complete and total acceptance of the patient's bizarre behaviour and symptomatology, without interfering with early interpretations of limit setting. The fostering of a positive relationship between therapist and patient was to be abandoned in later years when she focused her attention on the part played by hatred, aggression, anxiety and pathological splitting. Interpretation of transference, particularly of the negative, was considered a necessary element in the therapeutic process if malignant regression was to be avoided. Perhaps the greatest gift Fromm-Reichmann had was her overall attitude, verbal and non-verbal, with which she related to her patients, which conveyed a sense of courage, honesty, firmness, understanding and especially dedication; personal characteristics which formed the solid core upon which her technique was then built.

In 1947 Dr Dexter Bullard, Medical Director, called Dr Stanton, a psychiatrist, and Dr Schwartz, a sociologist, in order to set up a research of the social organisation of the hospital and of the effect that this structure had on the behaviour of patients and of hospital staff itself. The results of their three years rigorous research ${ }^{2}$ led to a major integration in the hospital approach: the understanding of the patient's internal world and of his interaction with the therapist was complemented with an emphasis of the importance of contextual issues. Stanton and Schwartz' unprejudiced and implacable review of the hospital organisation, of the roles of the professionals involved and those of the patients, and of the communicational aspects within the institution, broke down stereotypes entrenched in the hospital culture, and clearly pointed to the reflexive nature between certain symptomatic behaviour and dysfunctional social attitudes. For example, they showed that unrecognised chronic, intense disagreement (splitting) within two or more key members of the therapeutic team inevitably led to a worsening of the patient's mental state, which would improve once the splitting was brought into the open, properly addressed and resolved. 
In later years, important contributions were made by $\mathrm{Dr} \mathrm{Pao},{ }^{3} \mathrm{Dr}$ Will ${ }^{4}$ and others, but the name of Dr Harold Searles stands out. Searles, ${ }^{5}$ who stayed at Chestnut Lodge for nearly 14 years, describes transference and countertransference phenomena in great detail, as they emerged in his psychotherapeutic work with schizophrenic patients. He shows that a phase of 'therapeutic symbiosis'sets in during treatment, in which the analyst experiences and enacts the various transference roles projected into him by the patient.

\section{Hospital structure and therapeutic programme}

The hospital houses 85 adults suffering from schizophrenia, schizophreniform psychosis, major affective disorders, schizoaffective disorders and borderline states, 28 adolescents and 50 between out-patients and day patients. Most of them are chronically ill and have established themselves as treatment failures in previous institutions; this makes Chestnut Lodge a tertiary care hospital which treats patients in the poor prognosis spectrum. Hospital staff is made up of a pool of psychiatrists, nurses, social workers, recreational/occupational therapists and one senior psychologist. Hierarchically, the Medical Director is ultimately responsible for everything which happens within the hospital. The Clinical Director, aided by an Assistant Clinical Director, is responsible for every clinical matter both medical and non-medical. The Director of Psychotherapy has the function of assigning the newly admitted patients to a particular therapist in order to achieve the best match between the two; also, he is called upon whenever a dispute within the realm of the formal psychotherapy arises. The Director of Utilisation Review has the task of liaising with external bodies and institutions. The Director of Admissions assesses the patients who are referred to the hospital and allocates them to a particular unit. Also, there are two Directors of Research, a Director of the Adolescent Division, seven 'psychiatric administrators' (qualified psychiatrists) who have the responsibility for the patient's overall management on the ward, staff psychiatrists and third/fourth year residents. The therapeutic input includes several elements:

\section{(a) Individual psychotherapy}

All psychiatrists have a number of patients in individual psychodynamic psychotherapy, whose frequency ranges from two to six sessions per week throughout their stay at the hospital. Generally, this is continued on an out-patient basis after discharge. The theoretical orientation ranges from ego psychology derived approaches to views influenced by the interpersonal object relation theory as developed by Mahler, Sullivan, Fromm-Reichmann and Searles. Specifically, I would like to discuss two areas of their technique which differ from the work of most British psychoanalysts:

(i) the use of counter-transference feelings, and

(ii) the way the so-called 'real relationship' between the therapist and the patient is dealt with.

Some therapists at the Chestnut Lodge make use of a technical intervention consisting of reflecting back to and sharing with the patient the thoughts, feelings and fantasies evoked in them during the session, and at times they enact the patient's projections. In this stage, called the symbiotic phase, differences between analyst and patient cease to exist and the two are fused in a mutually dependent situation. Systematised delusions may be accepted and, to an extent, colluded with until such a time that the patient is ready to entertain less disturbed transferences.

The second point is strictly related to the first. The therapist often responds to the patient's request for establishing a 'more real relationship', which could involve the therapist to behave rather informally, engaging with the patient in social interactions. Both situations could be viewed as serious breaches of technique which gratify the patient's wishes for an equal relationship, which eliminates any difference between the patient and the therapist. The psychotic and borderline patient has severe difficulties in recognising the child part of himself engaged in a dependent relationship with a parental figure (therapist), which would become the source of great conflicts. The gratification of the patient's desire for a role reversal, which dislodges the therapist from his therapeutic standpoint, increases the existing ego weakness of the patient who cannot internalise and integrate the analyst as a strong, solid and un damaged object. However, the patient's high degree of disturbance and fragmentation may be very taxing for the therapist engaged in a long-term, daily relationship with them. The need for a greater modification of technique may stem from the intense and often unbearable countertransference feelings of despair, hopelessness and failure evoked in the therapist during the course of therapy.

\section{(b) Weekly group therapy}

This is a flexible option and the patient is selected for group attendance during his stay. The group experience is felt to be more relevant for the adolescents who are strongly encouraged to participate soon after admission.

\section{(c) Family therapy}

The patient's parents come for regular interviews with the ward social worker in order to explore their feelings in relation to the patient's disturbance and 
to create an atmosphere which will secure their co-operation while the patient is in hospital. Once a therapeutic alliance with the parents has been reached, and when the timing for the patient is felt to be right, a more formal family therapy is implemented.

\section{(d) Nursing}

This falls into two large categories:

(i) spending a large amount of time with the patient, which involves talking and listening to him, reading to and with him, discussing issues revolving around the patient's disabilities, treatment, and aiding him in various activities and situations;

(ii) attending to the patient's physical condition. These general supportive functions are carried out unobtrusively, and are tailored to the degree of the patient's psychopathology and stage of treatment. They are felt to enhance the patient's self-confidence and promote a feeling of achievement. The nurse in charge of the ward carries out a crucial role as coordinator within the ward and as intermediary between the ward staff, the psychiatric administrator and the therapist; also, she acts as a liaison between the ward and the rest of the hospital.

\section{(e) Medication}

About $80 \%$ of the patients are on psychotropic drugs. There is an ongoing debate within the hospital about this issue. Some believe that drugs reduce anxiety, regression and cognitive disorganisation, which render the patient more accessible to interpersonal contact. Others stress that medication is potentially capable of blunting affects and conflicts which are driven underground and hence would become inaccessible to psychodynamic work. In the absence of sound research on the interaction between psychotropic medication and psychotherapy, the general climate of USA psychiatry and the complicated medical-legal system are tipping the balance towards an increasing use of psychotropic drugs. Measures of physical containment such as seclusion rooms, cold sheet packs and physical holding are used in the event of severely violent behaviour or extreme psychotic agitation. Even in these circumstances, the therapist is expected to see his patient for daily sessions.

\section{(f) Unit meetings}

The ward team, made up of the psychiatric administrator, the charge nurse, staff nurses and the ward social worker, meets with the patient group at a variable weekly frequency (from one to three times according to the unit). The concession of privileges and other management issues are discussed, as well as acting out behaviours and any other aspects of the patient's life within and without the hospital. This is an arena in which patients often ventilate their hatred for the hospital and for the treating professionals, and good team cohesion and a great deal of tolerance is necessary in containing and working with aggression, manipulations and provocations.

\section{(g) Activities}

These include recreational and rehabilitative endeavours, dance and music therapy, psychodrama, etc. They are carried out by specialised professionals.

The hospital employs a 'bipolar' model, in which a distinction is made between the formal psychotherapeutic sphere and the sociotherapeutic sphere, the assumption being that the two realms are complementary but essentially different. In the individual psychotherapy the chaotic world of the patient is made sense of in terms of primitive internalised object relationships which are reactivated in the interaction with the therapist. The detailed interpretations of early pathological relationships with their drive derivatives, and of primitive defence mechanisms (pathological splitting, projective identification, manic denial) as they emerge in the transference are the vehicles for change and insight to develop. The hospital milieu stimulates social learning, promotes ego supportive activities, establishes emotionally significant relationships, encourages the taking on of responsibilities and allows the patient to experience a containing and holding environment.

These two areas are kept separate by a clearly defined boundary which ideally should neither become too rigid nor too permeable. The therapist is not part of the ward team, but communication between him, the psychiatric administrator and the charge nurse occurs according to the mutual necessities. This physiological splitting of functions is felt to be containing for patients whose original partial failure of splitting mechanisms did not allow them to make sense of the chaotic nature of their internal and external experiences, leading to the development of confusional states. However, the relationship between therapist, psychiatric administrator and charge nurse is regularly monitored by the therapist's own supervisor, in order to prevent the possible development of pathological splitting within hospital staff.

\section{Research}

Probably stimulated by the recurrent criticisms of the lack of scientific evidence in psychotherapeutic practice, Chestnut Lodge has devoted efforts and resources in the area of research in recent years. The team of the Chestnut Lodge Research Institute, led by Dr McGlashan, ${ }^{6,7}$ has carried out a long-term (averagely 15 years after discharge) follow-up study 
on $446(72 \%)$ patients treated between 1950 and 1975. The findings yield important information with regard to natural history, diagnosis and treatment efficacy. The study's retrospective design includes six elements to ensure methodological rigour (operation ally defined diagnostic criteria, adequate demographic/predictor characterisation of samples, outcome measured multi-dimensionally, independence of follow-up data collection from the diagnostic and demographic/predictor data collection, reliability testing of all measures, and bias testing of missing subjects sub-samples). These high standards make this study suitable for cross-study comparisons (important in the absence of a control group). Using eight different diagnostic systems, eight non-overlapping study diagnostic categories are defined, and three of them (schizophrenia, unipolar depression, borderline personality disorder) are subsequently compared on the various demographic/predictor and outcome variables. Of the Axis I disorders, patients with unipolar depression do well, while the bipolar sample is more difficult to judge because of its small size and atypical nature (the majority suffered from unipolar mania). The findings for the schizophrenic population are that only one in three patients has a moderate to good outcome, while the rest remain chronically ill or marginally functional at follow-up.

The Boston State Hospital and the 'Iowa 500' are the two long-term studies on chronic schizophrenic patients which stand out as suitable for cross-study comparison with the Chestnut Lodge findings. On outcome variables such as follow-up living situation, employment and social activity, the Chestnut Lodge sample do significantly better, when compared with the Boston State Hospital sample, even though possible differences in chronicity and/or socio-economic status between the two samples may account for the results. The 'Iowa 500' and the Chestnut Lodge outcomes are roughly comparable.

The fact that one out of three of these chronically disabled people respond to the treatment programme has led to further research ${ }^{8}$ to establish the possible presence of predictors of outcome and/or whether failure or success may be related to particular therapeutic interventions. The preliminary findings of the predictor analysis points to four variables which are central to the prediction of global outcome: family history of schizophrenia, premorbid interests and productivity, presence of some affect (depressed mood), and degree of psychotic assaultiveness.

The outcome for patients with borderline personality disorder shows that $80 \%$ of them are consistently improved on most outcome variables (employment, social activity, degree of psychopathology, global functioning). The borderline sample has similar results to the unipolar sample, and contrasting findings to the schizophrenic sample. These results are confirmed by the research carried out at the New York State Psychiatric Institute by Professor Stone. ${ }^{9}$ One interesting finding with the borderline group is that outcome varies as a function of time after discharge: the patient's global functioning is at its post-discharge peak after 10-19 years, whereas a gradual deterioration follows from then on.

Keats \& McGlashan ${ }^{10}$ have tried to build a typology of treatment processes through the longterm observation of single cases. They have been searching for clues as to what elements in the process of treatment might contribute to outcome. The strategy used is to elaborate an integrated body of conceptualisation about the various elements of the psychotherapeutic situation derived from an overview of the treatment as it has been applied and described over the years. Comparison of single case studies will enable comparison with what went on during therapy with such 'corpus' to determine the extent to which the technique understudy approximates to that which constitutes psychotherapy. In the operational definition of what constitutes psychotherapeutic treatment numerous elements and subelements are included, such as the nature of individual psychopathology and the nature of schizophrenia from the psychotherapist's point of view, elements of the treatment situation (patient, therapist, setting and process), general technical attitudes, general technical interventions (establishing a relationship, elucidating, tolerating, integrating, working through), special technical interventions with specific symptoms and resistances, and a discussion of mutative mechanisms. The study is still ongoing, but preliminary results seem to suggest that a substantial number of specific elements in the process of treatment must be present if a positive outcome is to be reached. Keats \& McGlashan are aware of the danger that such a collection of technical strategies might lend itself to cook-book concretisations, which they warn against, and they state that vital therapeutic elements, such as style and creativity, cannot be programmed or reviewed. Moreover, these general conceptualisations convey a great deal about the gestalt of the psychotherapeutic situation but they are not easily applicable to the single case owing to the inherent unpredictability and uniqueness of each therapeutic encounter.

\section{Comments}

Chestnut Lodge is one of the few psychiatric hospitals which has continued to adopt a psychodynamic approach to a variety of mental disorders. In recent years developments in the areas of sociotherapy, family therapy and psychopharmacology have been used as adjuncts to the modified psychoanalyticpsychotherapy which remains the cornerstone of 
the patient's treatment. Research has shown that depressed and borderline patients have better outcomes than schizophrenic patients. However, crossstudy comparisons demonstrate that the results obtained with schizophrenic patients are not inferior to the results obtained in centres using a predominantly biological approach.

\section{References}

${ }^{1}$ Fromm-Reichmann, F. (1950) Principles of Intensive Psychotherapy. Chicago: University of Chicago Press.

${ }^{2}$ Stanton, A. H. \& Schwartz, M. S. (1954) The Mental Hospital. London: Tavistock Publications.

${ }^{3}$ PAo P. N. (1979) Schizophrenic Disorders: Theory and Treatment from a Psychodynamic Point of View. New York: International University Press.
${ }^{4}$ WILL, O. A. (1975) The conditions of being therapeutic. In Psychotherapy of Schizophrenia (Ed. J. G. Gundisson). New York: Aronson. pp. 53-66.

${ }^{5}$ Searles, H. F. (1965) Collected Papers on Schizophrenia and Related Subjects. London: Hogarth Press.

${ }^{6}$ McGlashan, T. H. (1984) The Chestnut Lodge follow-up study (I and II). Archives of General Psychiatry, 41, 473-601.

7 _ (1986a) The Chestnut Lodge follow-up study (III). Archives of General Psychiatry, 43, 20-30.

8 - (1986b) The prediction of outcome in chronic schizophrenia. Archives of General Psychiatry, 43, 167-176.

${ }^{9}$ STONE, M. H. (in press) The natural history of borderline patients: I. Global Outcome. Psychiatric Clinics of North America.

${ }^{10}$ Keats, C. J. \& McGlashan, T. H. (1985) Intensive psychotherapy of schizophrenia. The Yale Journal of Biology and Medicine, 58, 230-254. 